\title{
Announcement of the Valid Publication of New Names and New Combinations Previously Effectively Published Outside the IJSB \\ List No. 1
}

The purpose of this announcement is to effect the valid publication of the following new names and/or combinations under the procedure described in Int. J. Syst. Bacteriol. 27(3):iv, 1977.

\begin{tabular}{|c|c|c|c|c|c|}
\hline \multirow[b]{2}{*}{ Name or combination } & \multirow[b]{2}{*}{ Proposed as: } & \multirow[b]{2}{*}{ Author(s) } & \multicolumn{2}{|c|}{$\begin{array}{l}\text { Literature cita- } \\
\text { tion(s) to: }\end{array}$} & \multirow[b]{2}{*}{ Nomenclatural type $^{a}$} \\
\hline & & & $\begin{array}{l}\text { Proposal } \\
\text { of name } \\
\text { or com- } \\
\text { bination }\end{array}$ & $\begin{array}{l}\text { Descrip- } \\
\text { tion of } \\
\text { the } \\
\text { taxon }\end{array}$ & \\
\hline $\begin{array}{l}\text { Desulfotomaculum ace- } \\
\text { toxidans }\end{array}$ & New species & $\begin{array}{l}\text { Widdel and } \\
\text { Pfennig }\end{array}$ & 6 & 6 & $\begin{array}{l}\text { Strain "Göttingen" } \\
(=5575=\text { DSM } 771)^{b}\end{array}$ \\
\hline Desulfuromonas & New genus & $\begin{array}{l}\text { Pfennig and } \\
\text { Biebl }\end{array}$ & 3 & 3 & $\begin{array}{l}\text { D. acetoxidans Pfennig } \\
\text { and Biebl }\end{array}$ \\
\hline $\begin{array}{l}\text { Desulfuromonas acetoxi- } \\
\text { dans }\end{array}$ & New species & $\begin{array}{l}\text { Pfennig and } \\
\text { Biebl }\end{array}$ & 3 & 3 & $\begin{array}{l}\text { Strain "South Orkney } \\
\text { Islands, Antarctic } \\
\text { Ocean" }(=11070= \\
\text { DSM 684) })^{b}\end{array}$ \\
\hline Eubacterium tarantellus & New species & Udey et al. & 5 & 5 & $\begin{array}{l}\text { Strain UM-87 }(=\text { ATCC } \\
2_{29255)^{b}}^{b}\end{array}$ \\
\hline Haemophilus segnis & New species & Kilian & 2 & 2 & $\begin{array}{c}\text { Strain HK316 } \\
\text { 10977 }^{b}\end{array}$ \\
\hline $\begin{array}{l}\text { Halobacterium saccharo- } \\
\quad \text { vorum }\end{array}$ & New species & $\begin{array}{l}\text { Tomlinson and } \\
\text { Hochstein }\end{array}$ & 4 & 4 & Strain M6 (=ATCC 29252) \\
\hline Spirochaeta halophila & New species & $\begin{array}{c}\text { Greenberg and } \\
\text { Canale-Parola }\end{array}$ & 1 & 1 & Strain $\mathbf{R S}^{c}$ \\
\hline
\end{tabular}

${ }^{a}$ Abbreviations: ATCC, American Type Culture Collection, Rockville, Md., U.S.A.; DSM, Deutsche Sammlung von Mikroorganismen, Göttingen, Germany; NCTC, National Collection of Type Cultures, London, U.K.

${ }^{b}$ Type designated by the author(s).

$c$ Type by monotypy.

\section{LITERATURE CITED}

1. Greenberg, E. P., and E. Canale-Parola. 1976. Spirochaeta halophila sp. n., a facultative anaerobe from a high-salinity pond. Arch. Microbiol. 110:185-194.

2. Kilian, M. 1976. A taxonomic study of the genus Haemophilus, with the proposal of a new species. J. Gen. Microbiol. 93:9-62.

3. Pfennig, N., and H. Biebl. 1976. Desulfuromonas acetoxidans gen. nov. and sp. nov., a new anaerobic, sulfur-reducing, acetate-oxidizing bacterium. Arch. Microbiol. 110:3-12.

4. Tomlinson, G. A., and L. I. Hochstein. 1976. Halobac- terium saccharovorum sp. nov., a carbohydrate-metabolizing, extremely halophilic bacterium. Can. J. Microbiol. 22:587-591.

5. Udey, L. R., E. Young, and B. Sallman. 1977. Isolation and characterization of an anaerobic bacterium, $E u$ bacterium tarantellus sp. nov., associated with striped mullet (Mugil cephalus) mortality in Biscayne Bay, Florida. J. Fish. Res. Board Can. 34:402409.

6. Widdel, F., and N. Pfenning. 1977. A new anaerobic, sporing, acetate-oxidizing, sulfate-reducing bacterium, Desulfotomaculum (emend.) acetoxidans. Arch. Microbiol. 112:119-122. 